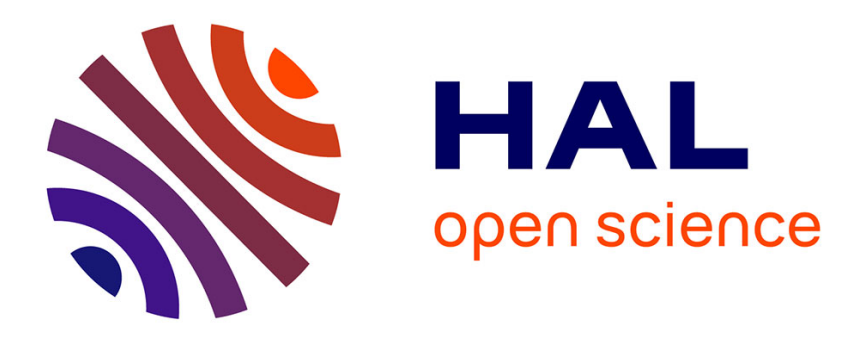

\title{
Les contradictions de la gestion intégrée des déchets urbains : l'incinération entre valorisation énergétique et refus social
}

Laurence Rocher

\section{- To cite this version:}

Laurence Rocher. Les contradictions de la gestion intégrée des déchets urbains: l'incinération entre valorisation énergétique et refus social. Flux - Cahiers scientifiques internationaux Réseaux et territoires, 2008, 2008/4 (74), pp.22-29. halshs-00685821

\section{HAL Id: halshs-00685821 \\ https://shs.hal.science/halshs-00685821}

Submitted on 6 Apr 2012

HAL is a multi-disciplinary open access archive for the deposit and dissemination of scientific research documents, whether they are published or not. The documents may come from teaching and research institutions in France or abroad, or from public or private research centers.
L'archive ouverte pluridisciplinaire HAL, est destinée au dépôt et à la diffusion de documents scientifiques de niveau recherche, publiés ou non, émanant des établissements d'enseignement et de recherche français ou étrangers, des laboratoires publics ou privés. 


\section{CA I R N}

chercher : repérer : avancer

Cet article est disponible en ligne à l'adresse :

http://www.cairn.info/article.php?ID_REVUE=FLUX\&ID_NUMPUBLIE=FLUX_074\&ID_ARTICLE=FLUX_074_0022

Les contradictions de la gestion intégrée des déchets urbains : I'incinération entre valorisation énergétique et refus social

\section{par Laurence ROCHER}

\section{Métropolis | Flux}

\section{8/4 - $\mathbf{N}^{\circ} 74$}

ISSN 1154-2721| pages 22 à 29

\section{Pour citer cet article :}

- Rocher L., Les contradictions de la gestion intégrée des déchets urbains : I'incinération entre valorisation énergétique et refus social, Flux 2008/4, N 74, p. 22-29.

Distribution électronique Cairn pour Métropolis.

(C) Métropolis. Tous droits réservés pour tous pays.

La reproduction ou représentation de cet article, notamment par photocopie, n'est autorisée que dans les limites des conditions générales d'utilisation du site ou, le cas échéant, des conditions générales de la licence souscrite par votre établissement. Toute autre reproduction ou représentation, en tout ou partie, sous quelque forme et de quelque manière que ce soit, est interdite sauf accord préalable et écrit de l'éditeur, en dehors des cas prévus par la législation en vigueur en France. Il est précisé que son stockage dans une base de données est également interdit. 


\title{
Les contradictions de la gestion intégrée des déchets urbains: I'incinération entre valorisation énergétique et refus social
}

\author{
Laurence Rocher
}

\begin{abstract}
es systèmes de gestion des déchets sont appelés à tendre vers Lune gestion dite intégrée, cette évolution étant déjà à I'œuvre à plusieurs titres. Nous proposons dans cet article un éclairage sur la politique française en matière de gestion des déchets ménagers, et notamment sur ses deux principes structurants que sont la valorisation (récupération des matières et de l'énergie) et la proximité (traitement des résidus au plus près de leur production). Ces principes, instaurés par la loi-cadre de 1992, font l'objet d'une traduction opérationnelle par les acteurs locaux à travers les choix des procédés de traitement et la définition d'un espace de gestion. Dans la mesure où ils reposent sur une inflexion des systèmes de traitement pour permettre la valorisation des matières et sur un ancrage territorial plus marqué des solutions de traitement, ces principes relèvent d'une intégration à la fois technique et territoriale. Cette intégration s'inscrit en adéquation avec les préceptes du développement urbain durable, en associant une gestion optimisée des ressources, une prise en compte « transversale » du problème et une responsabilisation des espaces urbains quant aux externalités qu'ils produisent.
\end{abstract}

L'incinération, qui est la destination de $43 \%$ des ordures ménagères générées en France en 2004 selon l'Ademe, permet une valorisation dite énergétique sous forme de chauffage urbain et/ou de production d'électricité. Il s'agit, à partir de cet exemple, de mettre en évidence les logiques de convergence et de divergence entre différentes composantes d'une gestion intégrée des déchets. Nous montrerons comment intégration technique et territoriale se rejoignent via une tendance qui se dessine à la requalification de la gestion des déchets à l'aune d'impératifs énergétiques, mais se heurtent au refus social de l'incinération. Les oppositions viennent en effet entraver l'implantation des équipements à proximité des espaces urbains, lieux des gisements des matières à éliminer et de réception de l'énergie produite. Elles viennent en outre forcer une mise en débat du problème, mettant à jour des points de tensions relatifs à la hiérarchisation entre différentes formes de valorisation, à la distinction déchet/ressource ou à l'équilibre fragile entre prévention/élimination/valorisation.

L'analyse est nourrie par une étude de la politique française de gestion des déchets ménagers ainsi que par des observations empiriques relatives à des processus de planification et des situations de conflits locaux (Rocher, 2006). L'attention est portée sur les processus de justification et de légitimation sur lesquels reposent les argumentations et positions des acteurs et institutions concernés, aux niveaux national et local. La thèse défendue est que la technique de l'incinération, qui répond dans une certaine mesure à une gestion intégrée des déchets mais suscite une opposition très forte, met en lumière les tensions entre les préceptes du développement durable appliqué à la gestion urbaine. La démonstration est organisée en trois temps. En premier lieu, un retour sur l'évolution de la politique française de gestion des déchets met en évidence la place de la valorisation et les outils adoptés pour la mettre en œuvre. Nous nous intéresserons ensuite aux ressorts de l'intégration territoriale vus à travers les attendus de la valorisation énergétique localisée, pour, dans un troisième temps, identifier les points de tension où vient achopper le couple intégration technique/intégration territoriale, lesquels viennent redéfinir les frontières du « problème déchets ».

\section{LES DÉCHETS, ENTRE RÉSIDUS À ÉLIMINER ET MATIÈRES PREMIÈRES À VALORISER}

La valorisation des matières a longtemps été la norme, au point qu'il n'existait pas de déchets voués à l'élimination, les résidus des activités domestiques et artisanales, autant de " matières premières urbaines » (Barles, 2005), étant dans leur totalité réintroduits dans les systèmes de production agricoles et industriels. 
Avec la rupture de cette complémentarité entre ville, industrie et agriculture autour de la période fin XIXe - début XXe apparaît la notion de déchet et la nécessité de se doter de moyens techniques et financiers pour en assurer l'élimination. Les villes optent alors pour l'incinération, procédé qui répond aux préceptes hygiénistes de l'époque, et offre l'espoir de faire perdurer la réutilisation des résidus, sous forme de chauffage urbain notamment. À cette période de "résistance »(1880-1920) décrite par Barles, succède celle du « renoncement » à la valorisation des sous-produits dont le traitement devient de plus en plus coûteux. Les résidus, longtemps dotés d'une valeur positive dans le cadre d'un recyclage généralisé, acquièrent une valeur négative, puisque l'élimination représente un coût pour les collectivités. Devant l'augmentation des volumes de matières et leur complexité croissante, l'élimination se généralise au cours du XXe siècle avec le recours à deux procédés: I'incinération et la décharge, sauvage puis contrôlée.

Dans les années 1970, la France se dote d'une politique publique dédiée à la gestion des déchets. Le texte fondateur de cette politique, la loi-cadre du 15 juillet 1975, est élaboré dans un contexte particulier, marqué par la crise pétrolière. La soudaine prise de conscience de la dépendance énergétique du pays est ressentie comme une menace très vive. Dans un souci de lutte contre le gaspillage d'énergie et de matières premières, les déchets tendent alors à être considérés comme une potentielle « matière première secondaire » (Bertolini, 1998). Le principe de valorisation est inscrit dans la loi de 1975: «L'élimination des déchets doit être assurée (...) dans des conditions propres à faciliter la récupération des matériaux, éléments ou formes d'énergie réutilisables » (loi n75-633, titre V, article 15), sans que pour autant se mette en place de manière systématique et à grande échelle un système de récupération et de recyclage. Les premières expériences locales de récupération de certains matériaux (papiers cartons, verre) apparaissent, souvent associées à des démarches caritatives. Mais la priorité est alors de généraliser les systèmes de collecte et d'élimination sur l'ensemble du territoire, notamment dans les espaces ruraux, la valorisation demeurant secondaire.

Il faudra attendre le vote de la seconde loi-cadre relative à la gestion des déchets (le 13 juillet 1992) pour que soit inscrite de manière plus formelle l'obligation « de valoriser les déchets par réemploi, recyclage ou toute autre action visant à obtenir à partir des déchets des matériaux réutilisables ou de l'énergie » (loi n92-646, article ler, 3e alinéa). Le développement de la récupération et de la valorisation résulte de la mise en place quelques mois auparavant (en avril 1992) du dispositif ÉcoEmballage. L'inscription de la valorisation dans la réglementation française a pris forme à travers la mesure emblématique de la loi de 1992: I'interdiction d'enfouir les " déchets bruts » à compter du 1er juillet 2002. Il s'agit de détourner des décharges les déchets qui n'ont pas fait l'objet d'une valorisation préalable, et de limiter l'enfouissement aux seuls « déchets ultimes » (1). L'interprétation de la notion de « déchets ultimes » est déterminante puisqu'il s'agit de la part des déchets restant après l'effort de valorisation. C'est aux acteurs locaux, dans le cadre des Plans départementaux notamment, qu'il revient de donner un sens au "déchet ultime », et donc de déterminer la nature et le degré de valorisation à atteindre avant mise en décharge. Rapidement cette mesure est interprétée comme la fin de la mise en décharge à l'échéance 2002, et contribue à un recours massif à l'incinération (Ademe, 1997), tandis que la contestation sociale, que l'on croyait ciblée sur la mise en décharge, se déplace de l'enfouissement vers l'incinération.

L'objectif de valorisation tel que prévu dans le texte de 1992 ne fait l'objet d'aucune obligation quantifiée, de même qu'il n'instaure aucune hiérarchie entre d'une part la « valorisation matière » qui consiste en la production de nouvelles matières premières à partir du recyclage des matériaux ou le compostage des matières organiques, et d'autre part la « valorisation énergétique ", c'est-à-dire la production d'électricité ou de chauffage urbain à partir de procédés thermiques dont le plus répandu est l'incinération. Devant l'augmentation des capacités d'incinération vont être introduits des objectifs de valorisation chiffrés (2) et des tentatives d'instaurer une hiérarchie ente les modes de valorisation. L'effort de valorisation qui s'est généralisé dans les années 1990 a porté essentiellement sur les emballages. Depuis plusieurs années maintenant, on tend vers une valorisation qui repose sur une approche par produits en fin de vie (pneus, véhicules hors d'usage, déchets d'équipements électriques et électroniques-DEEE). Cette évolution, qui découle notamment de la réglementation européenne, donne lieu à la production d'une réglementation spécifique par filière, dans une logique de responsabilisation des producteurs.

Outre les contraintes de nature réglementaire, le levier des aides financières est utilisé pour imposer des objectifs de valorisation, voire pour instaurer une hiérarchie entre les modes de valorisation. L'incinération n'est subventionnée qu'à la condition qu'elle inclue de la valorisation énergétique. Puis, à partir 
de 1996, I'aide à l'incinération versée aux collectivités par ÉcoEmballages est fonction des résultats obtenus en matière de recyclage et de compostage. Ainsi s'opère une différenciation entre les modes de valorisation: la valorisation énergétique doit être précédée d'une ou de plusieurs formes de valorisation matière. C'est en 1998, dans le cadre d'une circulaire de la ministre de l'environnement Voynet, qu'est fixé un objectif ambitieux de valorisation matière de $50 \%$, instaurant par là même une hiérarchie entre valorisation matière et valorisation énergétique. Cela dit, ce nouvel objectif, fixé à l'échelle nationale, donne lieu à des confusions quant à sa traduction dans les plans départementaux. Malgré ces inflexions, l'insuffisance des aides destinées au financement des collectes séparatives et du compostage, couplée à une absence d'incitation légale en matière de valorisation, ne permettront pas un engagement des collectivités vers des systèmes poussés de valorisation, au profit $d^{\prime}$ un report vers I'incinération et la valorisation énergétique, qui apparaissent comme des solutions de facilité (Buclet et al., 2000) alliant une haute fiabilité technique et une acceptabilité sociale supposée supérieure à la mise en décharge. Les projets d'installations d'incinération portés par les collectivités locales, alimentant des réseaux de chauffage urbain et/ou le réseau de distribution d'électricité, deviennent des « unités de valorisation énergétique ».

Les questions de la valorisation et de la hiérarchisation vont être renouvelées dès le tournant 2000 avec la montée en puissance des préoccupations en matière de changement climatique. Le secteur des déchets, producteur principalement de méthane et de $\mathrm{CO}_{2}$, estimé responsable de 3\% des émissions globales de GES (gaz à effet de serre) (MEDD, 2004), est invité à prendre part à l'effort national de réduction des émissions. L'enjeu climatique vient ainsi réactualiser le lien entre gestion des déchets et production d'énergie né avec le choc pétrolier des années 1970. La prise en compte des impacts atmosphériques et énergétiques des déchets prend un double sens: le secteur des déchets, appelé à participer à la réduction des émissions atmosphériques (notamment de méthane grâce à la récupération du biogaz des sites d'enfouissement), apparaît également pourvoyeur d'énergie alternative parfois qualifiée de renouvelable. "Avec la lutte contre le changement climatique, la récupération et la valorisation de l'énergie issue du traitement des déchets (incinération avec production de chaleur et d'électricité, méthanisation, biogaz de décharge) voient leur intérêt se renforcer. Les déchets font en effet partie de la biomasse et par- ticipent donc à la production d'énergies renouvelables. » (ADEME, 2002, p. 6).

L'abandon de l'idée de hiérarchisation entre types de valorisation prévaut alors au sein des sphères institutionnelles et professionnelles (3), conforté par le développement de méthodes d'évaluation de la pression environnementale qui promeuvent une appréhension globale des impacts telles que "I'analyse des cycles de vie »(ADEME/AMORCE, 2005). Le souci d'une évaluation environnementale globale intégrant la question des impacts atmosphériques est inscrit dans la réglementation relative à la planification de la gestion des déchets: à partir de 2005, les plans départementaux et régionaux d'élimination des déchets ménagers et industriels sont soumis à un rapport environnemental (4). Cette refonte de l'évaluation des impacts, marquée par l'attention portée aux émissions de GES, se heurte toutefois à des difficultés à circonscrire l'étendue de la mesure: doit-on, par exemple, prendre en compte les transports dans l'évaluation des impacts des systèmes de gestion des déchets?

La politique française de gestion des déchets s'efforce donc depuis les années 1970 de se démarquer d'une logique d'élimination pour aller vers une valorisation généralisée intégrant les enjeux énergétiques sous la pression de facteurs « exogènes » tels que l'indépendance énergétique puis les émissions de gaz à effet de serre. L'évolution des instruments incitatifs (négociation des tarifs d'achat de l'énergie, assujettissement des émissions de GES au Plan national d'affectation des quotas, aides aux équipements) s'avérera probablement déterminante dans la configuration des systèmes de gestion des déchets, notamment en matière d'intégration de la fonction de production énergétique. La réglementation européenne est d'autre part amenée à évoluer quant à la définition du statut de déchet et à la désignation des opérations d'élimination et de valorisation. Les systèmes locaux de gestion des déchets et la configuration technique des équipements, qui doivent répondre à un impératif de valorisation, doivent en outre s'inscrire dans une logique de proximité.

\section{LA PROXIMITÉ COMME PRINCIPE D'INTÉGRATION TERRITORIALE}

Depuis 1992, I'organisation du traitement des déchets doit répondre à un principe de proximité, dans le but de limiter le transport et ainsi de réduire des impacts environnementaux, 
mais également dans l'optique de renforcer « I'ancrage territorial », puisqu'il s'agit d'éliminer les déchets au plus près de leur lieu de production. L'inscription du principe de proximité est envisagée comme une réponse aux situations conflictuelles, considérées comme des manifestations de refus des nuisances générées par les "déchets des autres ». Ce faisant, il s'agit de faire coïncider le territoire de production au territoire de l'élimination, et de favoriser la « responsabilisation des citoyens visà-vis de leurs déchets » (Dron, 1997). Ce principe, censé s'appliquer à travers le découpage du territoire départemental en secteurs de collecte et de traitement dans le cadre des plans, n'est toutefois doté de valeur ni normative ni juridique. L'organisation territoriale de la gestion des déchets, fortement entravée par les difficultés d'implantation des équipements prévus, reste dominée par la répartition des équipements existants et les priorités de leurs gestionnaires (5). Devant la faiblesse des outils de planification et du principe de la "proximité », la recherche d'un territoire optimal et accepté pour la gestion des déchets demeure un objet de préoccupation des acteurs institutionnels et politiques. Le périmètre départemental étant jugé trop rigide, on lui préfère des formules plus souples telles que des " bassins d'activités ", des " communautés de destin » (CGP, 2003) ou encore des « territoires intermédiaires» (ADEME, 2005).

De nouveaux outils de gestion territorialisées répondant à une appréhension territoriale et transversale de la gestion des déchets voient le jour. Le "Contrat territorial déchets », " dispositif partenarial de gestion globale des déchets » est proposé depuis 1999 par l'Ademe aux collectivités volontaires selon une forme contractuelle et concertée. L'approche mise en avant relève d'une volonté affirmée d'intégration dans la mesure où elle vise une prise en compte la plus complète de l'ensemble de la chaîne de gestion des déchets, depuis la prévention jusqu'à I'organisation des services de collecte et de traitement. II s'agit de combiner une intégration intrasectorielle en " décloisonn[ant] la réflexion des différentes filières ", à une intégration intersectorielle allant vers une " corrélation entre la politique déchets et les autres thématiques constitutives de l'aménagement du territoire: développement de l'emploi, solutions locales, habitat, transport », dans l'« objectif d'inscrire la problématique déchets dans les politiques d'aménagement et de développement durable (?) »(ADEME, 2006). Les nouveaux outils de la gestion des déchets, rejoignant d'ailleurs en cela les attendus de la planification départementale, s'inscrivent en conformité avec les prescriptions de développement durable qui considèrent le niveau local comme le plus pertinent pour une approche transversale des problèmes (Theys, 2002).

L'intégration territoriale de la gestion des déchets, rendue effective avec les plans départementaux et réaffirmée par le biais de nouvelles démarches, va se trouver renforcée par la montée de l'enjeu énergétique, précisément par la volonté d'une appréhension territorialisée de la production énergétique. Les invitations à "'relocaliser' la production énergétique » (Sénat, 2006) s'accompagnent d'un regain d'intérêt à l'égard des réseaux de chaleur, parmi lesquels ceux alimentés par les ordures ménagères. Plus largement, la nécessité d'une planification locale énergétique (les rapporteurs du Sénat suggèrent l'élaboration de "schéma territorial des énergies locales et de la maîtrise de l'énergie ») encourage un couplage entre les secteurs des déchets et de l'énergie. II s'agirait ainsi de penser conjointement production énergétique et traitement des déchets dans le cadre de "schémas locaux de services publics, occasion d'une réflexion conjointe sur la gestion des déchets, l'aménagement du territoire et la production d'énergie » (Prévost, 2000).

Outre l'adéquation géographique et technique avec les gisements de déchets, les systèmes de gestion doivent ainsi être organisés au regard de leur fonction "en aval ", en l'occurrence des opportunités de valorisation. À l'objectif d'élimination selon des procédés respectueux de l'environnement et du principe de proximité entre gisement et traitement, s'ajoute celui d'une prise en charge des flux sortant, réaffirmé récemment à l'occasion du «Grenelle de l'environnement »: «(...) la conception des circuits de collecte et de traitement intermédiaire et ultime de tous les déchets doit être pensée à l'échelle des territoires en même temps que les usages du sol, les politiques énergétiques (pour la valorisation de ceux des déchets pouvant contribuer à la production énergétique) et celles de réduction des GES. » (Tuot, 2007, p. 21).

À ces multiples contraintes s'ajoute celle non moindre de l'acceptation des équipements, comprise comme leur intégration dans un environnement social. Or, loin d'apparaître en contradiction, ces différentes injonctions trouvent des points de convergence. Il s'agit par exemple de « Revaloriser l'image des sites de traitement à travers le développement parallèle de services rendus à la population et aux entreprises riveraines: vente de compost, livraison de chaleur, séchage du fourrage, chauffage des serres par exemple » (CGP, 2003, p. 532). La valorisation 
énergétique est envisagée comme un facteur d'acceptation locale des équipements, au point que la participation à la réduction des GES n'est plus prioritaire en tant que telle mais se présente comme un élément-phare de l'entreprise de justification de la solution. «Au plan national, il est intéressant de récupérer l'énergie contenue dans les déchets pour contenir les émissions de gaz à effet de serre mais l'enjeu reste modeste en termes de sécurité d'approvisionnement. Par contre, au plan local, I'utilisation de l'énergie contenue dans les déchets présente un grand intérêt. Si elle s'accompagne de la création d'activités nouvelles ou d'une offre intéressante de chauffage collectif, elle pourrait aider à faire accepter l'implantation des installations de traitement de déchets. Par ailleurs, si la récupération d'énergie est optimale, elle participera de façon très significative au financement du traitement des déchets: plus du tiers du coût de l'incinération dans les cas les plus favorables. » (Prévost, 2000, p. 18).

Les logiques de valorisation, de territorialisation et d'acceptation sociale s'imbriquent dans une reconfiguration de la politique publique de gestion des déchets qui s'efforce de se démarquer d'une logique d'élimination. Intégrations technique, territoriale et sociale se rejoignent pour renouveler le cadre de référence de la politique de la gestion des déchets. Pour autant, ce régime de justification va être mis à l'épreuve de controverses sur l'incinération, qui se manifestent à l'occasion de projets locaux.

\section{LES LIGNES DE FRACTURE DE L'INCINÉRATION OU L'ACCEPTATION SOCIALE EN ÉCHEC}

Alors que l'incinération, devenue valorisation énergétique, recherche un regain de légitimité en rejoignant une tendance à la territorialisation de la production d'énergie, le refus de cette technique tend à se radicaliser, comme en témoigne la revendication d'un moratoire à l'occasion des élections présidentielles de 2007 puis du « Grenelle de l'environnement ». L'objectif de valorisation locale se heurte au refus des populations de vivre à proximité de tels équipements, ce qui se traduit par la difficulté de connexion des incinérateurs aux réseaux de chauffage urbain. À ce titre sont d'ailleurs revendiqués des systèmes d'aide au transport de la chaleur issue de l'incinération ou de la combustion du biogaz, afin que l'extension des réseaux ne vienne pas pénaliser la rentabilité des projets (Sénat, 2006). Le refus social de l'incinération vient mettre à mal le rouage valorisation/territorialisation/acceptation, dans la mesu- re où la valorisation localisée peine à relever le défi de l'acceptation locale. Sur la base d'observations empiriques rassemblées à l'occasion d'une étude de terrain menée dans le département de I'Indre-et-Loire (Rocher, 2006), nous tentons de mettre en évidence les points de discordance entre les promoteurs et les opposants à cette technique, autant de points où se joue la définition des contours du "problème déchets ».

La place grandissante de l'argument climatique dans la gestion des déchets se traduit par des jeux de comptabilité des tonnes de $\mathrm{CO}_{2}$ évitées ou émises. Les collectivités locales et les gestionnaires privés mettent en avant les tonnes de $\mathrm{CO}_{2}$ évitées par un réseau de chaleur collectif comparativement à ce que représenterait l'équivalent des logements desservis par des chaudières individuelles. Les bénéfices induits en termes d'émissions justifient d'ailleurs l'exclusion des réseaux de chaleur, parmi lesquels les installations de traitement de déchets, du Plan national d'affection des quotas de GES. Les acteurs associatifs insistent en revanche sur l'incohérence de cette technique avec les objectifs de réduction des émissions de GES au regard des volumes de $\mathrm{CO}_{2}$ créés par la combustion. "L'incinération reste un mode d'élimination (...) Ce procédé réduit la taille des déchets en transformant la plus grande partie du carbone " capturé » sous forme solide en forme gazeuse. Ces dégagements gazeux contribuent grandement à la production de gaz à effet de serre sans rendement énergétique intéressant » (6).

Outre la question du $\mathrm{CO}_{2}$, d'autres sous-produits de l'incinération intervenant dans le bilan-matière du traitement des déchets donnent à voir la porosité de la frontière entre élimination et valorisation. C'est le cas des mâchefers: valorisés sous forme de sous-couches routières permettant d'économiser I'utilisation de granulats, ils permettent de tirer un bilan positif dans la balance entre déchets entrants et sous-produits valorisables. Considérés comme des déchets du traitement des déchets, auxquels s'ajoutent les résidus d'épuration des fumées d'incinération des ordures ménagères (REFIOM), le bilan déchets produits/déchets créés se trouve alors sinon inversé, du moins réduit. À travers ces luttes de définition entre élimination et valorisation, ce sont les frontières de la notion de déchet qui sont en cause.

À cela s'ajoute la question des risques et des différentes formes de pollution: les émanations atmosphériques, dont les dioxines sont les plus connues, mais aussi les substances contenues dans les mâchefers et dans les REFIOM qui doivent faire 
l'objet d'un traitement spécifique (enfouissement dans des centres de stockage de classe 1). La crainte de la dilution des polluants dans le milieu et dans le corps humain renvoie à une autre forme de la frontière du déchet (Van Staevel, 2005): I'incinération est apparentée à une machine à transformer des ordures inoffensives et circonscrites en des déchets particulièrement nocifs en raison de leur toxicité et de leur dissémination.

Appréhendée comme un maillon de la chaîne globale de la gestion des déchets, I'incinération est dénoncée au motif qu'elle entre en contradiction avec les objectifs affichés par ailleurs de réduction de la production, condamnant de fait la mise en place d'un système hiérarchisé privilégiant la valorisation matière. La combustion, outre qu'elle représente un gaspillage de matières premières, viendrait condamner tout espoir d'une valorisation matière poussée et de réduction à la source: en investissant dans des équipements coûteux, les collectivités sont engagées à fournir les déchets nécessaires au fonctionnement et à la rentabilité de l'équipement. Aussi, c'est en apportant une solution que cette technique empêcherait la mise en débat du problème et sa prise en charge en amont. C'est le recours à une solution porteuse d'une " clôture du débat » (Jobert, 1992; Fritsh, Ravon, 1993) qui viendrait entraver la mise en place d'une politique intégrée caractérisée par une prise en charge du problème dans sa globalité et de manière hiérarchisée.

Cette dissonance quant au statut de la solution se retrouve dans les préférences et les attentes en matière de configuration des équipements de part et d'autre des acteurs impliqués. Le traitement reposant sur une haute technicité - en l'occurrence, les incinérateurs - induit, pour des raisons de rentabilité économique, de fiabilité technique et environnementale, des équipements de grande taille desservant un vaste territoire. Les associations engagées contre de tels projets affichent en revanche une préférence pour des équipements offrant des critères d'accès et de réversibilité; " sites d'enfouissement réversible » ou "décharge provisoire » de petite taille, quitte à les multiplier. Outre les possibilités de surveillance et d'équité spatiale, il s'agit de garder ouvert le "problème déchets », condition d'une prise en compte de la production en amont et de la recherche de solutions alternatives, considérant que « la proximité permet de conserver une conscience sociétale du déchet » (7).

La valorisation localisée, en associant des objectifs d'intégration technique et territoriale, ne rencontre pas les attentes de ses promoteurs en matière d'acceptation sociale. Les arguments avancés contre ce procédé reposent sur une appréhension plus large de la question des déchets, placée au-delà des bénéfices et des impacts locaux. Cette construction argumentative, qui relève d'un mécanisme de "montée en généralité », participe d'une stratégie de contournement de la disqualification NIMBY qui menace les opposants à l'incinération. La valorisation énergétique, pourtant envisagée comme un facteur d'acceptation, est perçue comme une opération de verdissement d'une technique critiquée pour ses effets pervers en matière de gestion des déchets, et ses risques sanitaires et environnementaux.

\section{CONCLUSION}

À I'heure où la politique de gestion des déchets s'efforce de se démarquer d'une logique d'élimination au profit d'une valorisation plus poussée, les systèmes de gestion des déchets évoluent dans le sens d'une intégration qui rencontre les préceptes du développement durable, dès lors qu'ils s'appuient sur une approche transversale et globale, à la fois verticale et horizontale. Il s'agit de prendre en charge l'ensemble des " maillons de la chaîne ", du plus en amont, à travers des objectifs de prévention de la production des déchets, jusqu'en aval avec la gestion des impacts du traitement. Notons que les conditions économiques et techniques de cette intégration verticale sont pour une large part en débat (application de la « responsabilité élargie du producteur ", internalisation des externalités environnementales). À cette "intégration verticale » s'ajoute une "intégration horizontale » lisible d'une part, dans une relative redéfinition des frontières sectorielles de l'action publique locale et $d^{\prime}$ autre part, dans la recherche $d^{\prime}$ 'un ancrage territorial optimal. La gestion des déchets, dans la mesure où des objectifs de valorisation sont visés, tend à être pensée en fonction d'enjeux définis dans le cadre de politiques sectorielles connexes: la production énergétique comme on l'a vu, mais aussi par exemple la politique agricole pour certains types de déchets. Cette approche transversale localisée doit toutefois se combiner avec une certaine « sectorialisation » induite par la multiplication de filières spécifiques (pneus, DEEE, piles...) que nécessitent la complexité et la dangerosité des matériaux utilisés dans la fabrication des biens de consommation. Enfin, l'intégration territoriale repose sur l'édiction de principes d'action tels que le principe de proximité, ainsi que sur l'évolution des outils et cadres de l'action locale en matière de coopération intercommunale et de planification. 
L'exemple de l'incinération a permis de mettre en lumière des points de convergence ou au contraire de frottement entre plusieurs facettes d'une gestion intégrée. Le développement de la valorisation dans le cadre du choix français du recours à l'incinération d'une part, la montée des inquiétudes relatives au changement climatique d'autre part, ont conduit à une réactualisation du rapprochement entre les domaines de la gestion des déchets et de l'énergie. Ce rapprochement prend la forme d'une intégration de nature technique (la combustion doit être accompagnée d'une valorisation énergétique sous forme de production électrique, de chaleur ou de cogénération), associée à une intégration territoriale puisque cette valorisation doit répondre à une gestion territorialisée à la fois de la gestion des déchets et de la production énergétique.

Les motivations d'ordres technique et territorial convergent en faveur de systèmes intégrés de gestion des déchets, mais ne rencontrent pas les objectifs escomptés en matière d'acceptation sociale, dès lors que la technique de l'incinération rencontre une forte opposition des populations. Ces tensions mettent en lumière plusieurs points d'achoppement, relatifs au statut du déchet défini comme source d'énergie renouvelable ou matière à valoriser, aux contradictions entre les objectifs de la politique, à la quantification des impacts notamment en GES: autant de lignes de tensions où se jouent les contours du « problème déchets » saisi comme problème public. En outre, elles donnent à voir des visions et attentes divergentes quant à la configuration technique et territoriale des systèmes de gestion des déchets.
Ainsi, un hiatus demeure entre une approche privilégiant la sécurité environnementale, assurée par des équipements de grande taille dont la rentabilité soit suffisante pour développer des systèmes techniques de réduction des impacts, et une demande sociale de transparence qui passe par une réversibilité des choix et une répartition territoriale des équipements. À cela s'ajoute un hiatus relatif aux liens établis entre dimension sociale et proximité des équipements, entre une proximité envisagée comme facteur d'acceptation des solutions (les bénéfices de la valorisation sont alors considérés comme des compensations) et une proximité à même d'engendrer une saisie sociétale du problème à sa source.

Finalement, de la même manière que le développement durable, «mythe pacificateur » (Lascoumes, 1999) ayant pour " principale qualité de gommer les contradictions qu'il énonce »(Theys, 2002), les principes structurants de la gestion des déchets, la valorisation et la proximité, consensuels et partagés, font l'objet d'appropriations divergentes renvoyant à des luttes d'interprétation et des conflits de définition.

\footnotetext{
Laurence Rocher est auteure d'une thèse en aménagement de l'espace-urbanisme sur la gestion territoriale des déchets ménagers. Elle est actuellement chargée de recherche contractuelle à l'UMR CITERES, Université de Tours, où elle poursuit des travaux sur les politiques publiques d'environnement (gestion des déchets, changement climatique), et les conflits en milieu urbain. laurence.rocher@univ-tours.fr
}

\section{NOTES}

(1) «Est ultime au sens de la présente loi un déchet, résultant ou non du traitement d'un déchet, qui n'est plus susceptible d'être traité dans les conditions techniques et économiques du moment, notamment par extraction de la part valorisable ou par réduction de son caractère polluant ou dangereux », Loi n92646 du 13 juillet 1992. relative à l'élimination des déchets ainsi qu'aux installations classées pour la protection de l'environnement, article ler, 5e alinéa.

(2) Des objectifs quantifiés par types de matériaux (d'emballage), en application de la directive européenne de 1994, sont instaurés en 1996, puis révisés en 2005 (décret du 29 novembre 2005, résultant de la transposition de la « Directive emballages »). L'élimination des emballages doit donc être planifiée de manière spécifique suivant le principe d'une valorisation (y compris énergétique) à hauteur de $60 \%$ minimum en poids des déchets d'emballage et le recyclage de 55\% minimum, d'ici le 31 décembre 2008.

(3) En juillet 2000, le ministère de l'Économie publie un rapport sur La récupération de l'énergie issue du traitement des déchets. Les auteurs de ce document préconisent notamment de considérer au même titre la valorisation matière et la valorisation énergétique (qui consiste en la valorisation des biogaz issus du stockage et du compostage, de la chaleur issue de l'incinération), et ainsi d'orienter le choix des procédés de traitement en fonction de leur capacité de génération de matières premières énergétiques. " À l'origine de la politique de recyclage on trouve la volonté d'économiser des ressources non renouvelables; or un autre objectif est apparu, la maîtrise des émissions de gaz à effet de serre. À cet égard, il vaut mieux brûler le papier ou le carton et récupérer la chaleur que les recy- 
cler; il en est parfois de même du plastique. » (Prévost, 2000, p. 2).

(4) Décret 2005-1472 du 29 novembre 2005 modifiant le décret 96-1008 du 18 novembre 1996 relatif aux PDEDMA.

(5) L'instance d'évaluation du Commissariat Général du Plan, chargée d'une évaluation de la politique de gestion des déchets publiée en 2003, tire un bilan relativement négatif de l'application du principe de proximité, qui paraît « ne pas avoir fait le poids face aux principes de la libre administration des collectivités territoriales et, surtout, aux principes de concurrence et de libre circulation des marchandises. »(CGP, 2003, p. 54).

(6) Intervention de L.-C. Etile, Les Amis de la Terre, à I'occasion des 8es Assises Nationales des Déchets, septembre 2005 (Assises Nationales des Déchets, 2007).

(7) Ibid.

\section{BIBLIOGRAPHIE}

ADEME, 1997, Bilan des plans départementaux d'élimination des déchets et informations détaillées par département.

ADEME, 2002, "Dossier dix ans de politique déchets: premier bilan », La Lettre ADEME, $\mathrm{n}^{\circ} 20$.

ADEME/AMORCE, 2005, Comment évaluer les impacts environnementaux au moyen de l'analyse du cycle de vie.

ADEME, 2005, Déchets et territoires. De la planification à la gestion locale de tous les déchets. Le dossier du colloque, 22-23 juin, Paris.

ADEME, 2006, Contrat territorial déchets. Agir ensemble autrement.

Assises Nationales des DéCHets, 2007, 1993-2005. Les actes des Assises Nationales des Déchets.

Barles S., 2005, L'invention des déchets urbains. 1790-1970, Seyssel, Champ Vallon.

Bertolinı G., 1998, "La politique française de gestion des déchets depuis 1973 », In: Les politiques d'environnement. Évaluation de la première génération: 1971-1995, sous la direction de B. Barraqué et J. Theys, Paris, Recherches, pp. 171-188.

Bourg D., Buclet N. \& Gilotte L., 2003, Impact du risque technologique sur la stabilité institutionnelle d'un système organisationnel: le rôle de l'incinération dans la gestion des déchets ménagers et assimilés. Rapport final pour le Ministère de l'Écologie et du Développement Durable, Programme "Évaluation et prise en compte des risques naturels et technologiques ».

Buclet N., Defeuilley C., Lupton S., 2000, « Municipal waste management in France », In: Municipal waste management in Europe: a comparative study in building regimes, sous la direction de N. Buclet et O. Godard, Dordrecht, Kluwer, pp. 87-119.

Commissariat Général du Plan, 2003, Rapport de l'instance d'évaluation de la politique du service public des déchets ménagers et assimilés.

Dron D., 1997, Déchets municipaux. Coopérer pour prévenir. Rapport au ministre de l'environnement, Cellule Prospective et Stratégie.
Fritsch P., Ravon B., 1993, «Du problème en tant qu'il est à résoudre, au problème en tant qu'il est à débattre », In : Les raisons de l'action publique, CRESAL, Paris, L'Harmattan, pp. 339-347.

JOBERT B., 1992, "Représentations sociales, controverses et débats dans la conduite des politiques publiques ", Revue Française de Science Politique, vol. 42, n², pp. 219-233.

Lascoumes P., 1999, "Productivité des controverses et renouveau de l'expertise ", Les Cahiers de la sécurité intérieure, $\mathrm{n}^{\circ} 38$, pp. 75-95.

MEDD, 2004, Plan Climat 2004. Face au changement climatique agissons ensemble.

Prévost H., 2000, La récupération de l'énergie issue du traitement des déchets, Conseil général des Mines-Ministère de I'Économie, des Finances et de l'Industrie.

ROCHER L., 2006, Gouverner les déchets. Gestion territoriale des déchets ménagers et participation publique, Thèse de doctorat en aménagement de l'espace-urbanisme (dir. Larrue C.), Université de Tours, (http://tel.archives-ouvertes.fr/tel-00175228/fr)

SÉNAT, 2006, Énergies renouvelables et développement local. L'intelligence territoriale en action. Rapport $\mathrm{d}^{\prime}$ information $\mathrm{n}^{\circ} 436$ (2005-2006) de MM. Cl. Belot et J.-M. Juilhard.

THEYS J., 2002, " L'approche territoriale du « développement durable », condition d'une prise en compte de sa dimension sociale », Développement durable et territoire, Dossier 1: Approches territoriales du Développement Durable, mis en ligne le 23 septembre 2002.

(http://developpementdurable.revues.org/document1475.html)

TuOt T., 2007, Le Grenelle Environnement, Rapport du rapporteur général. disponible en ligne:

http://www.legrenelle-environnement.fr/grenelleenvironnement/spip.php?article530

Van Staevel E., 2005, "De l'extraordinaire ascension d'une molécule », In: Incinération des déchets ménagers: la grande peur, sous la direction de J. Brousse, Le Cherche Midi, pp. 111-127. 